\title{
Special focus: metals in medicine
}

\author{
John Spencer*,1 \& Ben Walden ${ }^{2}$ \\ ${ }^{1}$ Department of Chemistry, School of Life Sciences, University of Sussex, Falmer, Brighton, East Sussex, BN1 9QJ, UK \\ ${ }^{2}$ Future Science Group, Unitec House, 2 Albert Place, London N3 1QB, UK \\ * Author for correspondence: j.spencer@sussex.ac.uk
}

"Metals in biological systems hold key roles including facilitating enzyme function, oxygen transport and redox chemistry. As our understanding of the role of metals in the body improves, we can increasingly make use of their unique properties in the development of potential novel therapies."

First draft submitted: 22 December 2017; Accepted for publication: 3 January 2018; Published online: 6 March 2018

Keywords: cancer $\bullet$ cyclodextrins $\bullet$ ferrocene $\bullet$ metallodrugs $\bullet$ MRI $\bullet$ platinum

Metals in biological systems hold key roles including facilitating enzyme function, oxygen transport and redox chemistry. As our understanding of the role of metals in the body improves, we can increasingly make use of their unique properties in the development of potential novel therapies [1].

In the last half a century, metal complexes have become key to the treatment of cancer, making them some of the most commonly used therapeutics in the developed world. Cisplatin has proved unparalleled in its efficacy against tumors, interacting with DNA to trigger cell death [2]. The pioneering studies of Rosenberg, leading to the development of cisplatin as a form of treatment, came unexpectedly when his group found that platinum-based electrodes could inhibit strains of Escherichia coli [3]. Thanks to his relentless work in convincing industry to test platinum-based modalities against cancer, platinum drugs have become our most vital components in the fight against cancer over the last four decades and many clinical trials and combinations continue to be conducted [4].

However, since cisplatin's approval in 1978, few further metal based medicines have reached circulation. While considered by the WHO an essential drug, cisplatin has its flaws, prone to fast resistance build up in tumors, and with debilitating side effects through its cytotoxicity. Another problem lies in the lack of specificity of the active metal core, leading to undesirable biological activity and interactions with other biomolecules, although our improved understanding of such interactions may ultimately lead to more selective drugs [5].

In recent years, efforts have been made to combat these flaws. New mechanisms of delivery can improve the drug's specificity by extending ligand structures, facilitating transport to the site of action in cancerous cells. Prodrugs offer the opportunity to incorporate multiple motifs in a single complex, delivering several mechanisms of action together to combat the build up of resistance. This work has helped extend the interest of metallodrugs into other areas of medical research, ranging from antibiotics to the treatment of neurodegenerative diseases [6].

Hanif and Hartinger begin the issue by detailing cisplatin's flaws, and discussing the steps being taken to fix these problems [7]. Progress in precision medicine has brought new ways to selectively target cancer cells, and recently researchers have attempted to use metallodrugs to trigger immunomodulatory effects, reactivating the anti-cancer immune response. Yang then proposes a simple strategy for developing effective metallodrugs, giving platinum and vanadium-based examples [8]. He suggests that metallodrugs work through mimicking metal-regulated systems for signal transduction, and that drugs must incorporate 'enhancer' and 'antidote' structures, promoting signaling while preventing metal toxicity.

Koide and colleagues describe their initial work on improving the metallodrug carboplatin. Koide's team has begun incorporating an arginine-rich triple helical peptide motif into the complex [9]. The structure's positive charge can facilitate movement through cell membranes, releasing platinum under conditions found in the target location.

Not all research has focused on improving existing medicines. A short communication by Spencer and his team, as an extension to their earlier work in related areas [10], illustrates how ferrocene-derived structures could 
be used in the future to antagonize key GPCRs [11]. Ferrocene has long been of interest to medicinal chemists, with its favorable lipophilicity, lack of toxicity and capacity to generate reactive oxygen species making it an ideal drug candidate. Ferroquine, a ferrocene-based analog of chloroquine, has been effective in clinical trials against chloroquine-resistant malarial strains [12]. A number of other ferrocene analogs, such as ferrocifens [13], have shown promising anticancer action through their redox activity [14].

As an understanding of molecular recognition has improved, scientists have begun constructing structures to mimic the action of enzymes and important biomolecules. Cyclodextrin is an example of a structure of interest due to its hydrophobic cavity, which could be used to improve the bioavailability of lipophilic small molecules. In her joint review with Oliveri, Vecchio highlights recent efforts to incorporate cyclodextrin into metal complexes to build better therapeutic, diagnostic and theranostic drugs [15]. Indeed, while the therapeutic use of metal complexes has seen little success in the clinic, metals hold other positions of medical importance. MRI contrast agents, such as cardolite, are often now taken for granted; such is their impact in medicine and diagnosis. Gadolinium is key to MRI procedures and Chang discusses the development of new agents, their key features and problems [16]. More tailored complexes allow for more nuanced imaging, responding to different stimuli, and the review focuses on structures to identify blood pooling, enabling binding to specific organs or identify specific pathologies.

In their work, Lui et al. look to the future of metal-based medicines, targeting new classes of disease [17]. Alzheimer's disease manifests itself in the form of aggregates of amyloid- $\beta$ peptide, forming plaques in and around dendritic neurons. There is hope that metal chelators could be used therapeutically, coordinating to $\mathrm{Cu}$ (II), $\mathrm{Zn}$ (II) and $\mathrm{Fe}(\mathrm{III})$ residues in the peptide structure to prevent or reverse aggregation. Scaffolds binding to these metal cores could also aid the imaging of plaques, combining both properties for theranostic purposes.

It has been a pleasure to guest edit this exciting volume of Future Medicinal Chemistry, which brings together a broad variety of articles involving the use of metals in biological systems, and illustrates the potential for extending their functionality and scope not only in fundamental but also in applied science.

\section{Financial \& competing interests disclosure}

B Walden is an employee of Future Science Ltd. The authors have no other relevant affiliations or financial involvement with any organization or entity with a financial interest in or financial conflict with the subject matter or materials discussed in the manuscript apart from those disclosed.

No writing assistance was utilized in the production of this manuscript.

\section{References}

1 Biological Inorganic Chemistry. Bertini I, Gray H, Stiefel E, Valentine J (Eds). University Science Books, CA, USA (2007).

2 Wilson J, Lippard S. Synthetic methods for the preparation of platinum anticancer complexes. Chem. Rev. 114(8), 4470-4495 (2014).

3 Rosenberg B, Vancamp L, Krigas T. Inhibition of cell division in Escherichia coli by electrolysis products from a platinum electrode. Nature 205, 698-699 (1965).

4 Wallbillich J, Forde B, Havrilesky L, Cohn D. A personalized paradigm in the treatment of platinum-resistant ovarian cancer - a cost utility analysis of genomic-based versus cytotoxic therapy. Gynecol. Oncol. 142(1), 144-149 (2016).

5 Casini A, Mastrobuoni G, Temperini C et al. ESI mass spectrometry and x-ray diffraction studies of adducts between anticancer platinum drugs and hen egg white lysozyme. Chem. Commun. (Camb.) 14(2), 156-158 (2017).

6 Dabrowiak J. Metals in Medicine. John Wiley \& Sons Ltd, NY, USA (2009).

7 Hanif M, Hartinger C. Metallodrug research: where is the new cisplatin? Future Med. Chem. 10(6), 615-617 (2018).

8 Yang X. Regulating cellular stress responses: an emerging strategy for rational metallodrug design. Future Med. Chem. 10(6), 611-614 (2018).

9 Masuda R, Hayashi R, Nose H et al. Development of a carboplatin derivative conjugated with a collagen-like triple-helical peptide. Future Med. Chem. 10(6), 619-629 (2018).

10 Sansook S, Tuo W, Lemaire L et al. Synthesis of bioorganometallic nanomolar-potent CB2 agonists containing a ferrocene unit. Organometallics 35(19), 3361-3368 (2016).

11 Sansook S, Tuo W, Bollier M et al. Synthesis and biological evaluation of ferrocene-based cannabinoid receptor 2 ligands. Future Med. Chem. 10(6), 631-638 (2018).

12 Dive D, Biot C. Ferrocene conjugates of chloroquine and other antimalarials: the development of ferroquine, a new antimalarial. ChemMedChem 3(3), 383-391 (2008).

13 Jaouen G, Vessières A, Top S. Ferrocifen type anti cancer drugs. Chem. Soc. Rev. 44(24), 8802-8817 (2015). 
14 Patra M, Gasser G. The medicinal chemistry of ferrocene and its derivatives. Nat. Rev. Chem. 1, doi:10.1038/s41570-017-0066 (2017) (Epub ahead of print).

15 Oliveri V, Vecchio G. Metallocyclodextrins in medicinal chemistry. Future Med. Chem. 10(6), 663-677 (2018).

16 Chang Y, Kim H, Gang H. Gadolinium as an MRI contrast agent. Future Med. Chem. 10(6), 639-661 (2018).

17 Liu H, Qu Y, Wang X. Amyloid $\beta$-targeted metal complexes for potential applications in Alzheimer's disease. Future Med. Chem. 10(6), 679-701 (2018). 
